\title{
General practitioner notes as a source of information for case-control studies in young
} women

\author{
C E D Chilvers, M C Pike, C N Taylor, C Hermon, B Crossley, S J Smith, and the \\ UK National Case-Control Study Group
}

\begin{abstract}
Study objective - The UK National CaseControl Study was carried out to investigate the relationship between oral contraceptive use and breast cancer risk. This study investigates whether general practitioner notes could be used as the sole data source for epidemiological studies of young women and what the effect would be on non-response and recall bias.

Design - Case-control study with data on gynaecological, obstetric, and contraceptive history collected at interview and from general practitioners' notes. Information from these two sources was compared.
\end{abstract}

Setting - This was a population-based study.

Participants - Altogether 755 women with breast cancer aged under 36 years at diagnosis, each with an age-matched control, participated in the study. Response rates at interview were $72 \%$ and $89 \%$ for cases and controls but GP data were available for $90 \%$ of the 1049 case and first-selected control pairs.

Main results - There was generally good agreement between the two data sources with respect to obstetric history and gynaecological procedures (hysterectomy, oophorectomy, and tubal ligation). The use of intra-uterine devices, or diaphragm, and partner's vasectomy were not reliably recorded in the GP's notes. The overall results of the UK study would have been qualitatively the same with respect to the relationship between oral contraceptive use and breast cancer risk if GP notes only had been used, in spite of the fact that only about half of all oral contraceptive usage was recorded in the notes. Response rates would have been higher, recall bias eliminated, and the cost of the study halved.

Conclusions - When planning case-control studies in young women, the possibility of using GP notes as the primary data source should be considered. Lack of data on potential confounding factors is a possible drawback to such use. The practice of destroying GP's notes shortly after the death of patients seriously restricts the possibility of using these notes when studying rapidly fatal conditions.

( $\mathcal{f}$ Epidemiol Community Health 1994;48:92-97)
The UK National Case-Control Study Group was set up in 1983 to investigate the relationship between oral contraceptive use and breast cancer risk in young women. Sources of information included personal interviews and general practitioners' (GP) notes. The objectives of using GP notes were twofold: to supplement information relating to oral contraceptive brand and usage obtained at interview and as a means of checking for non-response bias and recall bias. ${ }^{1}$ Here we compare the information derived from interview with that abstracted from GP notes, evaluate certain aspects of the extent and nature of non-response and recall bias, and determine whether GP notes may be used as the sole data source for epidemiological studies in young women.

\section{Methods}

The UK National Case-Control Study has been described in detail elsewhere. ${ }^{1}$ Briefly, all women who were diagnosed as having breast cancer between 1 January 1982 and 31 December 1985 and who were resident in any of 11 health regions in the UK were included, provided that their breast cancer diagnosis was before their 36th birthday. For every case, one control subject was chosen, effectively at random, from the list of that case's GP. The control's date of birth was matched to within six months of the date of birth of the case, and the control had to have been registered with the GP before the date of diagnosis of the case. If a case could not be interviewed, no attempt was made to interview her matched control. If the chosen control could not be interviewed a second (or further) control was selected in the same manner. For both cases and controls, the study was further restricted to white women with no previous malignancy, severe mental handicap, or psychiatric condition. Every control was given a "pseudodiagnosis" date, the date on which she was exactly the same age as her matched case had been at diagnosis.

Personal interviews were carried out in the women's homes by trained interviewers. The same interviewer interviewed both members of each case-control pair. The interview included questions on basic demographic details, and reproductive and contraceptive history, including brands of oral contraceptives used. Questions were asked up to diagnosis or pseudodiagnosis date. After interview, data on obstetric and oral contraceptive history were abstracted from the GP notes by the same interviewers. Entries in the notes dated after 
the diagnosis/pseudodiagnosis date were ignored, even if they referred to events before that date. The notes were abstracted onto a structured form and interviewers were instructed to use all information (including correspondence) in the notes. Where an item in the notes was undated, the abstractor used her judgment as to whether it happened before or after diagnosis/pseudodiagnosis date by using the chronology of the notes. Other sources of information kept by the GP (for example the notes of a woman's husband or children) were not abstracted.

We wanted complete GP data on all eligible cases and their first selected controls, regardless of whether or not the interviews had been done. Where we were unable to interview a case, for whatever reason, permission was sought from the consultant and GP to abstract the information from her GP notes. Permission was sought from the GP, and if required by the GP, from the control also, to abstract the same information from her GP notes. If a first-selected control could not be interviewed for any reason, permission was also sought to abstract information from her GP notes even though a second (or subsequent) control had been successfully interviewed. Information was also sought from any family planning clinics that a woman recalled attending. The data from all sources were used to contruct the lifelong contraceptive calendar used for the main analyses of the study data. ${ }^{12}$

\section{STATISTICAL METHODS}

Agreement between the interview and the GP data was measured by means of the kappa statistic. ${ }^{3-5}$ The analyses of breast cancer risk using interview data alone and GP data alone were carried out using multivariate logistic regression methods for individually matched case-control studies. ${ }^{6}$ Relative risks were estimated by odds ratios. Significance levels quoted are two-sided.

Table 1 Comparison of medical and contraceptive history (excluding oral contraceptives) at interview and in general practitioner notes: 754 case-control pairs

\begin{tabular}{|c|c|c|c|c|c|c|}
\hline & $I+$, & $I-, N-$ & $I+, N-$ & $I-, N+$ & Agreement & Kappa† \\
\hline \multicolumn{7}{|c|}{ Hysterectomy: } \\
\hline Cases & 11 & 742 & 1 & 0 & $753 / 754(99.9 \%)$ & 0.956 \\
\hline Controls & 9 & 741 & 2 & 2 & $750 / 754(99 \cdot 5 \%)$ & $0.816 \ddagger$ \\
\hline All & 20 & 1483 & 3 & 2 & $1503 / 1508(99.7 \%)$ & $0.887^{+}$ \\
\hline \multicolumn{7}{|c|}{ Oophorectomy: } \\
\hline Cases & 8 & 746 & 0 & 0 & $754 / 754(100 \%)$ & 1.000 \\
\hline Controls & 7 & 744 & 1 & 2 & $751 / 754(99 \cdot 6 \%)$ & $0.822 \ddagger$ \\
\hline All & 15 & 1490 & 1 & 2 & $1505 / 1508(99.8 \%)$ & 0.908 \\
\hline \multicolumn{7}{|c|}{ Tubal ligation: } \\
\hline Cases & 59 & 687 & 7 & 1 & $746 / 754(98.9 \%)$ & 0.931 \\
\hline Controls & 67 & 676 & 10 & 1 & $743 / 754(98 \cdot 5 \%)$ & 0.916 \\
\hline All & 126 & 1363 & 17 & 2 & $1489 / 1508(98.7 \%)$ & 0.923 \\
\hline \multicolumn{7}{|c|}{ IUD use, ever: } \\
\hline Cases & 132 & 554 & 55 & 13 & $686 / 754(91 \cdot 0 \%)$ & 0.739 \\
\hline Controls & 154 & 547 & 44 & 9 & $701 / 754(93.0 \%)$ & 0.808 \\
\hline All & 286 & 1101 & 99 & 22 & $1387 / 1508(92.0 \%)$ & $0 \cdot 774$ \\
\hline \multicolumn{7}{|c|}{ Diaphragm use, ever: } \\
\hline Cases & 13 & 677 & 58 & 6 & $690 / 754(91.5 \%)$ & 0.259 \\
\hline Controls & 15 & 658 & 75 & 6 & $673 / 754(89 \cdot 3 \%)$ & 0.236 \\
\hline All & 28 & 1335 & 133 & 12 & $1363 / 1508(90 \cdot 4 \%)$ & 0.247 \\
\hline \multicolumn{7}{|c|}{ Partner's vasectomy: } \\
\hline Cases & 48 & 611 & 93 & 2 & $659 / 754(87 \cdot 4 \%)$ & 0.449 \\
\hline Controls & 36 & 634 & 84 & 0 & $670 / 754(88.9 \%)$ & 0.419 \\
\hline All & 84 & 1245 & 177 & 2 & $1329 / 1508(88 \cdot 1 \%)$ & 0.436 \\
\hline
\end{tabular}

* $\mathrm{I}+=$ recalled at interview; $\mathrm{I}-=$ not recalled at interview; $\mathrm{N}+=$ recorded in general practitioner notes; $\mathrm{N}-=$ not recorded in general practitioner notes.

+ All $\mathrm{p}<0.001$.

$\ddagger$ Test for difference in agreement between cases and controls: $p<0.01$.
Results

RESPONSE RATES

Response rates and reasons for non-response have already been reported. ${ }^{1}$ Of the 1049 eligible cases, $755(72 \%)$ were interviewed and GP notes were abstracted for 754 of these 755 . GP notes were also abstracted for $222(76 \%)$ of the 294 cases not interviewed. Reasons for not abstracting the remaining 72 sets of notes were consultant or GP refusal (35), case refusal (5), and failure to trace the notes (32).

Of the 755 first-selected controls, $675(89 \%)$ were interviewed and the GP notes of 674 of these were abstracted. The 80 first controls not interviewed were replaced by second or subsequent choices and GP notes were abstracted for these also. Fifty three (66\%) of the 80 firstselected controls who were not interviewed also had their GP notes abstracted. Reasons for not abstracting the remaining 27 sets of notes were GP refusal (15), control refusal (11), and failure to trace (1). We also abstracted 219 sets $(99 \%)$ of notes for firstselected controls matched to the 222 noninterviewed cases whose notes had been successfully abstracted. (Subsequent controls were substituted for the three remaining control women who refused permission for note abstraction.)

We compared information obtained from interview with that obtained from GP notes for the 754 pairs where both sets were available; one GP refused permission for abstraction. GP notes were abstracted for a total of $946(90 \%)$ of the 1049 case and first-selected control pairs.

COMPARISON OF INFORMATION OBTAINED FROM GENERAL PRACTITIONER NOTES AND AT INTERVIEW

Agreement on whether a woman had ever been married was $86.0 \%$ (1297 of 1508) overall, and similar for cases and controls. In 204 instances the woman reported at interview that she had been married at that time but there was nothing in the GP notes to indicate that she had ever been married. Seven women reporting themselves never married were recorded as ever-married in the GP notes. The notes of these seven women were rechecked; two of the women reported themselves as cohabiting at interview and one of these had had two previous names in the GP notes. For four women the GP notes implied that they were married, and one was an error.

Agreement between interview and GP notes for medical and contraceptive history (excluding oral contraceptives) was at least $85 \%$ for all items (table 1). Agreement, as measured by the kappa statistic, varied between 0.24 and 1.00 with a good agreement on surgical procedures, and much poorer recording of contraceptive use in the GP notes. There was little difference between the rates of agreement for cases and controls, but certain aspects of the results clearly warranted further investigation. Some $87.0 \%$ (20 of 23 ) of hysterectomies and $93.8 \%$ (15 of 16) oophorectomies reported at interview were recorded in the GP notes. Two 
Table 2 Comparison of obstetric history from interview and general practitioner notes: 754 case-control pairs

\begin{tabular}{llllll}
\hline & \multicolumn{3}{l}{ General practitioner notes } & \\
\cline { 2 - 4 } & More & Agree & 1 less & >1 less & Kappa* \\
\hline Live births: $\dagger$ & & & & & \\
Cases & 18 & 689 & 26 & 21 & 0.906 \\
Controls & 17 & 683 & 34 & 20 & 0.904 \\
All & 35 & 1372 & 60 & 41 & 0.905 \\
Stillbirths: & & $(91.0 \%)$ & & & \\
Cases & 3 & 747 & 4 & 0 & 0.784 \\
Controls & 2 & 747 & 5 & 0 & 0.755 \\
All & 5 & 1494 & 9 & 0 & 0.770 \\
Miscarriages: & & $(99.1 \%)$ & & & \\
Cases & 12 & 689 & 44 & 9 & 0.762 \\
Controls & 18 & 700 & 31 & 5 & 0.829 \\
All & 30 & 1389 & 75 & 14 & 0.795 \\
Terminations: & & $(92.1 \%)$ & & & \\
Cases & 17 & 720 & 16 & 1 & 0.866 \\
Controls & 15 & 723 & 15 & 1 & 0.791 \\
All & 32 & 1443 & 31 & 2 & 0.834 \\
& & $(95.7 \%)$ & & & \\
\hline
\end{tabular}

* Weighted kappa. All $p<0.001$ except for stillbirths among controls where $p=0.003$

$\dagger$ In GP notes, pregnancy with outcome unknown assumed to be live birth.

control women recorded in their GP notes as having had both an (undated) hysterectomy and an oophorectomy did not report their surgery at interview and it is possible that it was carried out after the pseudodiagnosis date. A total of 126 of $143(88 \cdot 1 \%)$ tubal ligations reported at interview were also recorded in the notes. Two recorded in the notes had been reported at interview as taking place after diagnosis. Altogether 286 of 385 (74.3\%) selfreported users of intrauterine devices (IUD) and $17.4 \%$ (28 of 161 ) of self-reported diaphragm users had their use recorded in the notes. Twenty two women recorded in their GP notes as having used an IUD and 12 recorded as having used a diaphragm did not recall such use at interview. Partner's vasectomy recalled at interview was recorded in only $32 \cdot 2 \%$ ( 84 of 261 ) of the women's notes. The two instances where a vasectomy was recorded in the notes, but not at interview, arose because of discrepancies in dating in relation to diagnosis.

Agreement between interview and GP notes for various aspects of obstetric history was generally good (table 2; all weighted kappa statistics between 0.76 and 0.91 and all $p<0.001$ except for stillbirths among controls where $p=0.003$ ). There tended to be underrecording of obstetric events in the notes, apart from termination of pregnancy; 164 terminations were recalled and reported but an additional 40 were recorded in the GP notes. Thus the rate of under-reporting was at least $19 \cdot 6 \%$. We investigated further the records of the 35 women for whom more livebirths were recorded in their notes than were reported at

Table 3 Number of additional known brands of oral contraceptive recalled at interview by number of additional known brands recorded in general practitioner notes

\begin{tabular}{|c|c|c|c|c|c|c|c|c|c|c|}
\hline \multirow{3}{*}{$\begin{array}{l}\text { No of } \\
\text { additional } \\
\text { known brands } \\
\text { recorded in } \\
\text { notes }\end{array}$} & \multicolumn{10}{|c|}{ No of additional known brands recalled at interview } \\
\hline & \multicolumn{5}{|c|}{ Cases } & \multicolumn{5}{|c|}{ Controls } \\
\hline & 0 & 1 & 2 & $\geqslant 3$ & Total & 0 & 1 & 2 & $\geqslant 3$ & Total \\
\hline $\begin{array}{l}0 \\
1 \\
2 \\
\geqslant 3\end{array}$ & $\begin{array}{r}257 \\
75 \\
34 \\
32\end{array}$ & $\begin{array}{r}107 \\
71 \\
37 \\
18\end{array}$ & $\begin{array}{r}47 \\
25 \\
11 \\
9\end{array}$ & $\begin{array}{r}19 \\
9 \\
2 \\
1\end{array}$ & $\begin{array}{r}430 \\
180 \\
84 \\
60\end{array}$ & $\begin{array}{r}264 \\
83 \\
48 \\
14\end{array}$ & $\begin{array}{r}112 \\
67 \\
25 \\
21\end{array}$ & $\begin{array}{l}49 \\
24 \\
11 \\
13\end{array}$ & $\begin{array}{r}12 \\
8 \\
1 \\
2\end{array}$ & $\begin{array}{r}437 \\
182 \\
85 \\
50\end{array}$ \\
\hline Total & 398 & 233 & 92 & 31 & 754 & 409 & 225 & 97 & 23 & 754 \\
\hline
\end{tabular}

interview. Our assumption that a pregnancy with an unknown outcome in the notes was most likely to be a livebirth was wrong for 15 pregnancies (13 miscarriages and two terminations). One baby not reported at interview had been adopted and one reported as a neonatal death was a miscarriage. For one case a letter reporting a pregnancy filed in the notes referred to another woman. We identified six errors but could not explain 11 discrepancies.

Approximately half the total duration of oral contraceptive use recalled at interview was recorded in GP notes. Where a mention of oral contraceptive use was recorded without a duration or prescription, two alternative assumptions were made: that each mention referred to six or three cycles of use (these being the two most common prescriptions). Making the assumption that a mention was equivalent to a six cycle prescription, the mean durations recalled at interview and reported in the notes were 64.2 months and 35.1 months, respectively, for cases and 53.9 and 29.9 months, respectively, for controls. Making the assumption of three cycle prescriptions, the mean duration reported in the notes were 31.2 months and 26.2 months for cases and controls respectively.

Table 3 cross-tabulates the number of additional brands of oral contraceptive recorded in the GP notes but not recalled at interview, and the number of additional brands recalled at interview but not recorded in the notes. There was agreement between interview and GP notes with respect to known brands for $34.5 \%$ (521 of 1508) of women. A total of 138 women had no use of oral contraceptives either reported at interview or recorded in their GP notes. Nine women had a prescription in their GP notes but had never actually taken the pills prescribed. (This was established by re-contacting the women concerned and the total of 147 never-users corresponds with our previous report.) ${ }^{1}$ The data from cases and controls were comparable, the percentages with agreement on brands being $34.1 \%$ and $35.0 \%$ respectively. Some women reported use of oral contraceptives but could not recall the brand, and use of oral contraceptives of unknown brand was also recorded in the GP notes. These episodes of use have not been included in the above analysis.

\section{COMPARISON OF RELATIVE RISKS OF BREAST} CANCER IN RELATION TO ORAL CONTRACEPTIVE USE USING INTERVIEW AND GP DATA

In the main analyses of these data, combined data from the questionnaire and GP notes were used (see the first and second columns of table 4). ${ }^{12}$ The combination of data from the two sources (supplemented by use of family planning clinic records) effectively allowed us to identify most of the unknown brands used. We have now also calculated odds ratios using the interview data alone and the GP data alone (see third and fourth columns of table 4); similar results were obtained whichever dataset was used. This result is at first consideration surprising since only about half the information 
Table 4 Comparison of relative risks of breast cancer by duration of oral contraceptive (OC) use using data from general practitioner notes alone, interview alone, and combined data

\begin{tabular}{|c|c|c|c|c|c|}
\hline \multirow{2}{*}{$\begin{array}{l}\text { OC use } \\
\text { (mth) }\end{array}$} & \multicolumn{2}{|c|}{ Interview and GP notes } & \multirow{2}{*}{$\frac{\text { Interview }}{\text { Unadjusted }}$} & \multicolumn{2}{|l|}{ GP notes } \\
\hline & Unadjusted & Adjusted* & & 754 pairs unadjusted $\dagger$ & 946 pairs unadjusted $\dagger$ \\
\hline 0 & 1.00 & 1.00 & 1.00 & 1.00 & 1.00 \\
\hline $1-48$ & 0.90 & 0.95 & 0.93 & $1 \cdot 10$ & $1 \cdot 14$ \\
\hline 49-96 & 1.31 & 1.43 & $1 \cdot 18$ & 1.50 & 1.38 \\
\hline $97+$ & 1.66 & 1.74 & 1.69 & 1.74 & 1.90 \\
\hline
\end{tabular}

* Adjusted for age at menarche, nulliparity, age at first full-term pregnancy, breast feeding (ever, never), and family history of breast cancer.

$\dagger$ Assuming mention $=6$ months. recalled at interview is in the GP notes. This would bias the GP results upwards but is effectively compensated for by the random error in using the GP notes. When the GP data are analysed for the 946 cases each matched to their first selected control the odds ratios are slightly higher than for the 754 interviewed pairs.

\section{Discussion}

Agreement between information obtained from GP notes and at interview was good for surgical procedures (hysterectomy, oophorectomy, and tubal ligation) but less good for contraceptive use: partner's vasectomy was relatively rarely reported in the woman's notes and there was under-recording of IUD and of diaphragm use. The under-recording of contraceptive use is partly due to the use of nonGP family planning clinics. Differences in agreement between cases and controls were generally small, although for oophorectomy and hysterectomy the agreement was better for cases than controls.

There was "almost perfect" (see Landis and $\mathrm{Koch}^{7}$ ) agreement between the obstetric information obtained at interview and recorded in the notes for six of 12 items (that is, kappa statistics greater than $0 \cdot 80$ ). Termination of pregnancy was as likely to be under-reported as it was to be under-recorded. This was in spite of very careful questioning on pregnancy and its outcome, and our awareness that under-reporting of terminations of pregnancy would be likely to occur. In practice, as the GP notes are unlikely to record all terminations, the rate of under-reporting at interview will be higher than shown in table 2.

The GP notes varied enormously in quality with, at one extreme, impeccable recording of all items including contraception, and at the other, notes that must be regarded as inadequate. It may well be that in some instances notes had been "lost" on registration with a new GP, but it would still be reasonable to expect that basic information on obstetric and medical history might be recorded at a first visit to a new doctor. Changes in the contracts for general practitioners are likely to lead to more consistency and completeness in GP notes.

Studies comparing information recorded in medical records and recalled at interview have generally had the objective of validating the information recalled, ${ }^{8-14}$ but the premise that medical records are the gold standard for comparison has also been questioned. ${ }^{1516}$ The re- sults of studies comparing retrospective recall with information collected prospectively by participants, ${ }^{1718}$ with family planning clinic records, ${ }^{9}$ or hospital records ${ }^{12}$ have been reviewed by Harlow and Linet. ${ }^{19}$ More recently comparisons between hospital records, postal questionnaires, and interviews with respect to information on pregnancy and childbirth, ${ }^{15}$ and of records with interview reports of ovarian surgery ${ }^{20}$ have been made.

Rates of agreement in our study for oophorectomy and hysterectomy were similar to those reported previously. ${ }^{1014} \mathrm{~A}$ comparison of recall of surgical history using a postal survey with GP records showed that respondents provided accounts which were more reliable than GP records. ${ }^{21}$

Reproductive history has been compared with antenatal records ${ }^{1215}$ and with prospectively collected personal records, ${ }^{18}$ neither of which are directly comparable to our data. Similar results $(93 \%$ agreement on number of children compared to our $91.0 \%$ ) were found in the retirement community study where history was taken at entry rather than being recorded contemporaneously in the GP notes. ${ }^{10}$

Studies which have compared oral contraceptive use in medical records with recalled information have generally included only selfreported users of oral contraceptives, ${ }^{891113}$ although one study did include reported nonusers. ${ }^{22}$ Agreement on various parameters of oral contraceptive use was generally good. The questionnaire used in our study ${ }^{1}$ (which included a contraceptive calendar and album of photographs of oral contraceptive packaging) has been compared with an earlier questionnaire which included only a list of brand names. ${ }^{13}$ The former was demonstrably better for total duration of use but results were similar for other parameters. The women chosen had all used oral contraceptives yet when a comparison with GP notes was made, $32 \%$ of notes had no mention of oral contraceptive use and only $32 \%$ of changes of brand were recorded. It would be expected that concordance in the study reported here ${ }^{1}$ would be better because oral contraceptives were prescribed by both GPs and family planning clinics, whereas in the earlier comparison ${ }^{13}$ contraceptives were obtained only from family planning clinics. Although GPs are notified of clinic prescriptions it is clear that these are not always filed in the notes. The similarity of cases and controls with respect to agreement between interview and GP notes, and numbers of additional brands reported suggests that recall by cases is no better than controls (see also discussion in UK national study ${ }^{1}$.

The main results from the UK national study were based on a combination of data from the two sources and from family planning clinic records with re-interviews where there were major differences or inconsistencies between the data sources. ${ }^{1}$ Our finding that only $50 \%$ of recalled use of oral contraceptives is recorded in the notes, is in agreement with the conclusion of Coulter et $a l^{13}$ that GP records are insufficient for obtaining a complete 
oral contraceptive history. Yet, essentially we obtained the same relative risk result using the GP notes alone (table 4). A previous casecontrol study of malignant melanoma ${ }^{22}$ also reported consistent relative risks for ever-use of oral contraceptives from GP records and postal questionnaire, and a case-control study of risk of liver cancer $^{23}$ in relation to oral contraceptive use using GP notes alone had results compatible with those obtained in a questionnaire based case-control study. ${ }^{24}$ There were, however, certain methodological difficulties in the latter study (different questionnaires administered and different distributions of usual place of residence for cases and controls).

It may be argued that in the same way that the interviewers could not be blind to the casecontrol status of interviewees, it was not possible for them to be blind to case-control status when carrying out note abstractions. We felt that the only way to deal with any possible bias was firstly by having highly structured questionnaires and secondly by carefully training the interviewers. GP notes are laborious to extract and our interviewers reported that the concentration needed to abstract notes that may be disorganised and voluminous is such that case-control status becomes an irrelevance.

Using notes alone would have had a major impact on response rates. The response rates for cases would have been much greater: $93 \%$ (976 of 1049) versus $72 \%$ (755 of 1049) and as the major cause of inability to abstract notes was failure to trace them, the rate would be even higher had there been fewer delays in identifying cases. (Untraced notes usually belonged to deceased cases and $16.6 \%$ of cases had died before they could be interviewed.) The abstraction rate for first-selected controls was $97 \%$ (946 of 977). The main reason for failure to abstract control notes was that the control women who refused interview were less likely to give consent for note abstraction. If an interview had not been requested the abstraction rate would have been higher: the notes of $219(99 \%)$ of the 222 first-selected controls for non-interviewed cases where an interview was not requested were successfully abstracted. Permission to abstract GP notes was always sought at interview from cases and controls. Where cases and controls were not interviewed, permission was only obtained where individual GPs required it. Disclosure of information from notes for medical research does not require the explicit consent of the patient provided that confidentiality is scrupulously safeguarded..$^{25} \mathrm{It}$ is clear that at the time that this study was carried out response rates would have been higher using GP notes than for the interview study and potential nonresponse bias would be reduced and recall bias eliminated. Written permission for note abstraction is, however, now required much more often than during the mid-1980s, and the response rates for controls might now be similar to the $89 \%$ for the interview study. Whether the same proportion of women who were successfully interviewed would be willing to give permission to have their notes abstracted only is not known. If the approach to them was made through their GP it is likely, in our opinion, to be the same or even higher than the $89 \%$ that we obtained for the interview study. Using GP notes only, the cost of the study would be reduced by at least $50 \%$. The major disadvantage of using notes alone would be the lack of information on potential confounding factors. In our main analyses of risks associated with oral contraceptive use we adjusted the odds ratios for age at menarche, nulliparity, age at first full-term pregnancy, breastfeeding (ever, never), and family history of breast cancer. Of these factors, information on parity and age at first full-term pregnancy would be available from the notes. We did not investigate the frequency with which family history of breast cancer in a first degree relative was recorded in the GP notes. In our study the adjusted odds ratios were only slightly higher than the unadjusted odds ratios (table 4) but it would be unwise to assume that this would always be the case.

In conclusion, we could have carried out a much less expensive study of the association between oral contraceptive use and breast cancer risk and would still have found a strong positive trend in risk with increasing duration of use. The magnitude of the risk estimates would, however, be imprecise because, on average, only about half of the oral contraceptive use recalled was recorded in the notes. A "validation" sub-study could, however, be used to adjust the results obtained from GP notes, and this possibility should be further explored. Information on potential confounding factors would have been insufficient for an adjusted analysis to have been carried out and this must be regarded as an important limitation on studies using GP notes.

In this paper we have shown the value of GP notes for epidemiological research. In Britain the system whereby primary care notes follow an individual, in theory at least, from the cradle to the grave, is unique. At present, the Department of Health recommends that Family Health Services Authorities retain records for seven years after death and then destroy them. In practice, this interval may be reduced because of lack of storage space. A valuable resource is then lost. With the computerisation of general practice notes it is unclear to what extent a paper copy of computerised information will be stored in the notes passed on should a patient re-register elsewhere. If paper copies (of prescriptions, for example) were to be routinely stored in the notes, then it is likely that information would be more reliably recorded than at present. If not, there will be a deterioration in the completeness of data relevant to future research.

Other principal investigators in this study were: Klim McPherson (Department of Public Health and Policy, London School of Hygiene and Tropical Medicine), J Peto (Section of Epi-
demiology, Institute of Cancer Research, Sutton, Surrey), M P Vessey (Department of Public Health and Primary Care, University of Ortment

The UK National Case-Control Study was funded by the Imperial Cancer Research Fund, the Cancer Research CamImperial Cancer Research Fund, the Cancer Research Cam-
paign and the Medical Research Council. SJS is supported by paign and the Medical Research Council. SJS is supported by CEDC was Visiting Professor at the Department of Social and 
Preventive Medicine, University of Queensland. We thank Dr Christopher Bain and Dr Christopher Del Mar for helpful suggestions and Vineta O'Malley and Melanie Cumpston for manuscript preparation.

1 UK National Case-Control Study Group. Oral contraceptive use and breast cancer risk in young women. Lancet 1989;i:973-82.

2 UK National Case-Control Study Group. Oral contraceptive use and breast cancer risk in young women: subgroup analyses. Lancet 1990;335:1507-9.

3 Cohen J. A coefficient of agreement for nominal scales. Educational and Psychological Measurement 1960;20:3746.

4 Cohen J. Weighted Kappa: nominal scale agreement with provision for scaled disagreement or partial credit. Psychol Bul 1968;70:213-20.

5 Fleiss JL, Cohen J. The equivalence of weighted kappa and the intraclass correlation coefficient as measures of reliability. Educational and Psychological Measurement 1973;33:613-9.

6 Breslow NE, Day NE. Statistical methods in cancer research. The analysis of case-control studies (IARC Sci Publ no 32). Lyon: International Agency for Cancer Research, 1980.

7 Landis JR, Koch GG. The measurement of observed agreement for categorical data. Biometrics 1977;33:159-74.

8 Stolley PD, Tonascia JA, Sartwell PE, Tockman MS, Tonascia S, Rutledge A, Schinnar R. Agreement rates between oral contraceptive users and prescribers in relation to drug use histories. $A m \mathcal{F}$ Epidemiol

9 Glass R, Johnson B, Vessey M. Accuracy of recall of histories of oral contraceptive use. Br F Prev Soc Med 1974;28:273-5.

10 Paganini-Hill A, Ross RK. Reliability of recall of drug usage and other health-related information. Am $\mathcal{F}$ Epidemiol 1982;116:114-22

11 Rosenberg MJ, Layde PM, Ory HW, Strauss LT, Bourne Rooks J, Rubin GL. Agreement between women's histories of oral contraceptive use and physician records. Int $\mathcal{f}$ Epidemiol 1983;12:84-7.

12 Tilley BC, Barnes AB, Bergstralh E, et al. A comparison of pregnancy history recall and medical records: impli- cations for retrospective studies. Am $\mathcal{J}$ Epidemiol 1985;121:269-81.

13 Coulter A, Vessey M, McPherson K, Crossley B. The ability of women to recall their oral contraceptive histories. Contraception 1986;33:127-37.

14 Colditz GA, Stampfer MJ, Willett WC, Stason WB, Rosner B, Hennekens CH, Speizer FE. Reproducibility and validity of self-reported menopausal status in a prospectvalidity of self-reported menopausal status in a prospec

15 Oakley A, Rajan L, Robertson P. A comparison of different sources of information about pregnancy and childbirth. $\mathcal{f}$ Biosoc Sci 1990;22:477-87.

16 McKinney PA, Alexander FE, Nicholson C, Cartwright RA, Carrette J. Mothers' reports of childhood vaccinations and infections and their concordance with general practitioner records. F Public Health Med 1991;13:13-22.

17 Bean JA, Leeper JD, Wallace RB, et al. Variations in the reporting of menstrual histories. Am $\mathcal{J}$ Epidemiol 1979;109:181-5.

18 Wilcox AJ, Horney LF. Accuracy of spontaneous abortion recall. Am $\mathcal{F}$ Epidemiol 1984;120:727-33.

19 Harlow SD, Linet MS. Agreement between questionnaire data and medical records. Am f Epidemiol 1989;129:233 48.

20 Irwin KL, Wingo PA, Lee NC. Agreement of self-reported ovarian number following gynecologic surgery with medivarian number following gynecologic surgery with

21 Coulter A McPherson K, Elliott S, Whiting B. Accuracy of recall of surgical histories: a comparison of postal survey recall of surgical histories: a comparison of postal survey data and genera

22 Adam SA, Sheaves JK, Wright NH, Mosser G, Harris RW, Vessey MP. A case-control study of the possible association between oral contraceptives and malignant melanoma. Br F Cancer 1981;44:45-50.

23 Forman D, Vincent TJ, Doll R. Cancer of the liver and the use of oral contraceptives. $B M Y$ F 1986;292:1357-61.

24 Neuberger J, Forman D, Doll R, Williams R. Oral contraceptives and hepatocellular carcinoma. $B M \mathcal{F}$ 1986;292:1355-7.

25 Medical Research Council. Responsibility in the use of personal medical information for research. London: Medical Research Council, 1985. 